\title{
Identification of Class I Methanol Masers with Objects of Near and Mid-Infrared Bands and the Third Version of the Class I Methanol Maser (MMI) Catalog
}

\author{
Olga Bayandina, Irina Val'tts and Grigorii Larionov \\ Astro Space Center of the Lebedev Physical Institute, Moscow, Russia \\ email: bayandix@yandex.ru
}

\begin{abstract}
An identification has been conducted of class I methanol masers with 1) short-wave infrared objects EGO (extended green objects) - tracer bipolar outflow of matter in young stellar objects, and 2) isolated pre-protostellar gas-dust cores of the interstellar medium which are observed in absorption in the mid-infrared in the Galactic plane. It is shown that more than $50 \%$ of class I methanol masers are identified with bipolar outflows, considering the EGO as bipolar outflows (as compared with the result of $22 \%$ in the first version of the MMI catalog that contains no information about EGO). 99 from 139 class I methanol masers (71\%) are identified with SDC. Thus, it seems possible that the MMI can be formed in isolated self-gravitating condensations, which are the silhouette of dark clouds - IRDC and SDC.
\end{abstract}

Keywords. Masers, catalogues, etc.

\section{MMI CATALOG DESCRIPTION}

The catalog is a table in electronic form at http://www.asc.rssi.ru/MMI with a file 'readme' and a file of references (101 ref items). In our analysis, the identification was carried out by comparing the equatorial coordinates of sources. Since class I methanol masers usually were observed with a single dish with a beam of about $2^{\prime}$, identified sources were considered if the distance between the coordinates did not exceed $2^{\prime}$.

\section{RESULTS OF STATISTICAL ANALYSIS}

Identification was made with a help of TOPCAT (http://www.star.bris.ac.uk/ mbt/ topcat/) for the catalogues presented in:

206 MMI - http://www.asc.rssi.ru/MMI

$>300$ objects EGO - http://vizier.cfa.harvard.edu/viz-bin/VizieR?-source=J/AJ/ 136/2391 (Cyganowski et al. 2008),

10931 objects IRDC - http://vizier.cfa.harvard.edu/viz-bin/VizieR?-source=J/ApJ/ 639/227 (Simon et al. 2006),

11303 objects SDC - http://vizier.cfa.harvard.edu/viz-bin/VizieR?-source=J/A+A/ 505/405 (Peretto \& Fuller 2009).

Stastistical estimates were made for 206 class I methanol masers (MMI), except for identification with EGO and SDC, because only 139 MMI fall in the longitude interval of Spitzer. Results: in star-forming regions class I methanol masers have been identified

- in $42 \%$ of cases (59 sources from 139) with EGO; 
- in $16 \%$ of cases (33 sources from 206) with infrared dark clouds IRDC from MSX;

- in $71 \%$ of cases (99 sources from 139) with infrared dark clouds SDC from Spitzer.

\section{CONCLUSIONS}

- The third version of the class I methanol maser MMI/SFR catalog was compiled and presented in the Internet: http://www.asc.rssi.ru/MMI.

- 206 class I methanol maser were catalogued detected mostly at $44 \mathrm{GHz}$ in the direction of well-known star-forming regions (MMI/SFR) until the end of 2011 (results from Chen et al. 2011 - EGO survey at $95 \mathrm{GHz}$ in 2011 - were not included).

- Many methanol maser sources are objects of mixed type, combining classification features of both classes.

- In the last version of the catalog more than $50 \%$ of class I methanol masers are associated with bypolar outflows - if outflows traced by EGO.

- It is shown that only $33 \mathrm{MMI}$ are identified with IRDC from MSX survey, but for SDC (from Spitzer), this number increases to 99 , that is $71 \%$ of MMI.

- Thus, it seems possible that MMI can be formed in the isolated self-gravitating condensations, which at certain stages of evolution could be IRDC/SDC. Sample of SDC may be a new list for to study in order to detect new MMI. It is noteworthy that this work has been done positive in the direction of IRDC in the center of the Galaxy (see Deguchi et al. 2011).

Support for this work was provided by Russian Foundation of Basic Research (project number 10-02-00147-) and Federal National Scientific and Educational Program (project number 16.740.11.0155).

\section{References}

Chen, X., Ellingsen, S. P., Shen, Z-Q., Titmarsh, A. et al. 2011, ApJSS, 196, Issue 1, article id.9 Cyganowski, C. J., Whitney, B. A., Holden E., Braden, E. et al. 2008, Astron. J., 136, 2391

Deguchi, S., Tafoya, D., Shino N. et al. 2011, arXiv1109.0677D

Peretto, N. \& Fuller, G. A. 2009, A\&A 505, 405

Simon, R., Jackson, J. M., Rathborne, J. M., Chambers, E. T. et al. 2006, ApJ, 639, 227 